\title{
Digital Communication using Chaos and Nonlinear Dynamics
}

\author{
Lucas Illing \\ Reed College, Portland, OR 97202
}

\begin{abstract}
Digital communication using synchronized chaos is reviewed on the example of an optoelectronic transceiver and a wireless transceiver that utilizes chaotic pulse position modulation. Challenges that arise due to noise and other distortions occurring in the communication channel are pointed out and ways to improve the noise characteristics of the synchronization and to make chaos communication robust enough to work with realistic channels are discussed.
\end{abstract}

Key words: chaos, synchronization, chaos communication, optoelectronic, pulse position modulation

2008 MSC: 37D45, 94A14, 78A60

\section{Introduction}

Starting in the 1990s research on the chaotic dynamics of nonlinear system has focused on the problem of chaos control [1], on the topic of chaos synchronization $[2,3,4]$ and on ideas for possible applications of chaos. One such application is chaos communication, where one utilizes the chaotic signals that are generated by nonlinear circuits or optical systems as carriers for information transmission. The simplicity of many chaos generators and the rich structure and broadband nature of chaotic signals are the most attractive features that caused significant interest in possible chaos communication schemes.

In order to place chaos communication in context, a simplified block diagram of a typical traditional digital communication system that transmits binary data is presented in Fig. 1. Each block in the transmission chain performs a unique function, with modulation and demodulation being the only essential steps; all other processing steps are design options that can be used 


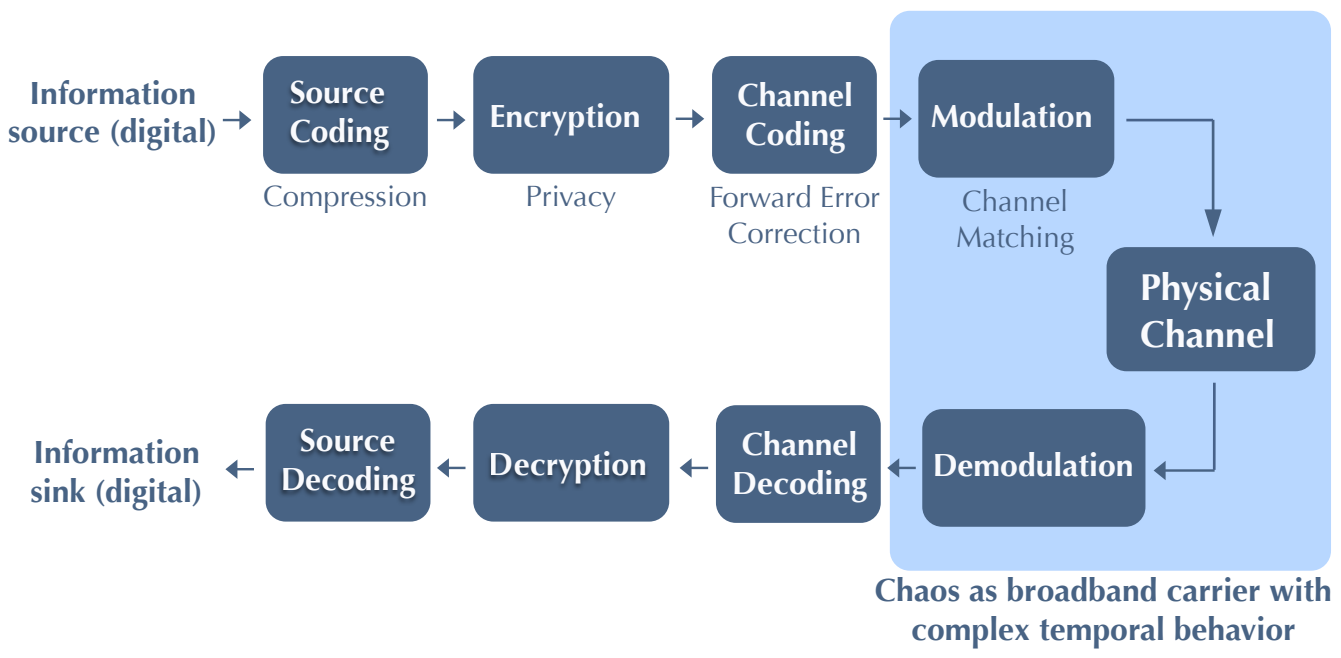

Figure 1: Simplified block diagram of a digital communication system.

to address application specific needs [5]. The incoming digital data is first compressed by the source coding block such that bandwidth is not wasted by transmitting redundant information. The encryption block re-codes the data in order to enhance transmission security. The channel encoding performs further transformations on the input data to minimize the degradation of the information bearing signal due to channel impairments. It takes into account the particular modulation scheme that is used and the characteristics of the channel through which transmission is to take place. Modulation impresses the data onto an analog radio-frequency (or optical frequency) carrier resulting in waveforms compatible with the transmission channel. Finally, for multiple-access applications, this signal may be combined with other signals (not shown) before it is transmitted through the physical channel, e.g. send through an optical fiber or radiated from an antenna. Each block in the receiver chain performs the inverse operation to that of the transmitter chain. The advantage of digital communications over traditional analog techniques is the excellent and independent control over security, bandwidth utilization, and error rate.

Although nonlinear techniques can be applied to all blocks in the system, we will focus here on the chaotic modulation of digital data onto a chaotic carrier as indicated in Fig. 1. Potential advantages of using nonlinear devices for digital communication schemes include improved efficiency [6, 7], lower power consumption, lower probability of intercept, and lower probability of 
detection [8]. These potential advantages have to be balanced against potentially lower performance in terms of bit-error rates, the need for efficient spectrum management, and the cost of implementing this new technology alongside the traditional infrastructure.

The purpose of this contribution is to provide a brief overview of digital chaos communication on the example of an optical and a wireless communication scheme. Interested readers can find a more extended and in-depth discussion in Larson et al. (2006) [9].

\section{Synchronization based chaos communication using optoelectronic devices}

Most traditional communication schemes are based on periodic signals and carrier waveforms stored at the receiver. Because chaotic carrier waves are nonperiodic they cannot be stored in the receiver as a reference to achieve coherent detection of the transmitted signal. One approach to address this problem is to transmit, along with the modulated signal, the unmodulated original chaotic waveform either using a separate channel or using time division [10]. In this approach reliable detection can be achieved at the expense of an at least $3 \mathrm{~dB}$ loss in the signal-to-noise-ratio. Another method is to encode the message into the symbolic dynamics of the chaotic transmitter $[11,12,13]$, with the result that the information can be read off simply by observing the transmitted signal. However, this method requires detailed and precise control of the transmitter dynamics, which is an extremely challenging task especially in high-speed optical systems. For this reason essentially all optical chaos communication schemes are based on the regeneration of the chaotic carrier at the receiver by means of chaos synchronization.

Though the synchronization of clocks (periodic systems) has been studied with great care over centuries, it was the surprising discovery of temporal synchronization between two chaotic systems that initiated the field of chaotic communications. The crucial realization was that, when two chaotic systems are coupled to each other in a suitable way, it is possible for a chaotic system in the receiver to follow the time evolution of an identical chaotic system in the transmitter, even when the chaotic systems start from very different initial conditions $[2,3,4]$. Therefore a chaotic signal generated in the transmitter can be replicated identically in the receiver in a stable manner. Communication based on chaotic synchronization was already proposed by Pecora and Carroll (1990) [4] in their seminal paper on chaos synchroniza- 


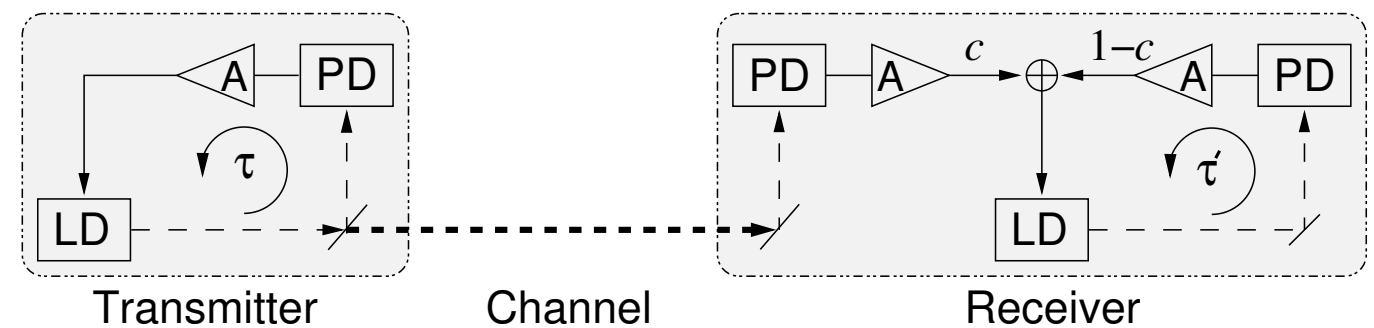

Figure 2: Schematic of the setup used to investigate the synchronization of two chaotic semiconductor lasers with delayed optoelectronic feedback. LD: laser diode; PD: photodetector; A: amplifier; $\tau$ : feedback delay time.

tion and developed further by several authors [14, 15, 16, 17]. The feasibility of chaos communication using synchronization was confirmed experimentally soon thereafter in electronic circuits [18, 19] and laser systems [20, 21, 22].

As a concrete example of optical systems that exhibit identical chaos synchronization consider the two coupled optoelectronic devices shown in Fig. 2, which were investigated theoretically by Abarbanel et al. (2001) [23] and experimentally by Tang and Liu (2001) [24]. The transmitter and receiver each consist of a single-mode semiconductor laser with amplified and time-delayed feedback formed by an optoelectronic loop from the laser's optical output back to the bias current across the laser itself. The transmitter and receiver generate chaotic oscillations at gigahertz frequencies due to the interplay of laser nonlinearities and time-delayed feedback, which makes the state-space of the system formally infinite-dimensional.

To achieve synchronization, the systems are unidirectionally coupled as shown in Fig. 2, where a fraction of the transmitter's chaotic output intensity is detected, amplified and coupled into the driving current of the receiver with coupling strength $c$. If $c=1$, there is no feedback signal in the receiver and we call this open loop. If $c=0$, the transmitter and receiver are completely decoupled. The equations of motion describing the time-delay dynamics of the transmitter-receiver system read

$$
\left\{\begin{array}{l}
\frac{d s_{T}}{d t}=\gamma_{\mathrm{c}}^{T}\left\{\left[g\left(n_{T}, s_{T}\right)-1\right] s_{T}\right\}+2 \sqrt{s_{T}} F_{T}^{s p} \\
\frac{d n_{T}}{d t}=\gamma_{\mathrm{s}}^{T}\left\{\mathcal{J}-n_{T}-\mathcal{J} g\left(n_{T}, s_{T}\right) s_{T}\right\}+\gamma_{\mathrm{s}}^{T} \xi\{\mathcal{J}+1\} s_{T}(t-\tau),
\end{array}\right.
$$




$$
\left\{\begin{aligned}
\frac{d s_{R}}{d t}=\gamma_{\mathrm{c}}^{R}\left\{\left[g\left(n_{R}, s_{R}\right)-1\right] s_{R}\right\}+2 \sqrt{s_{R}} F_{R}^{s p} \\
\frac{d n_{R}}{d t}=\gamma_{\mathrm{s}}^{R}\left\{\mathcal{J}-n_{R}-\mathcal{J} g\left(n_{R}, s_{R}\right) s_{R}\right\} \\
\quad+\gamma_{\mathrm{s}}^{R} \xi\{\mathcal{J}+1\}\left\{c s_{T}(t-\tau)+(1-c) s_{R}\left(t-\tau^{\prime}\right)\right\}
\end{aligned}\right.
$$

Here, $s$ denotes the dimensionless intracavity photon density, which is proportional to the measured output intensity of the laser, $n$ denotes the dimensionless carrier density, which quantifies the population inversion in the laser gain medium, and $g(n, s)$ specifies the gain ${ }^{1}$. The laser is pumped by an external dc-bias current proportional to $\mathcal{J}$, the photon survival time in the laser cavity is quantified by the photon decay rate $\gamma_{c}$, and the rate of carrier decay (mainly due to spontaneous emission) is given by $\gamma_{\mathrm{s}}$. The parameter $\xi$ denotes the feedback strength, $\tau$ is the feedback delay, and $F^{s p}$ is a white Gaussian noise term that models the contributions of spontaneous emission to the lasing mode.

For the case of an ideal channel and identical lasers without noise $\left(\gamma_{\mathrm{c}}^{T}=\right.$ $\gamma_{\mathrm{c}}^{R}, \gamma_{\mathrm{s}}^{T}=\gamma_{\mathrm{s}}^{R}, \tau=\tau^{\prime}$, and $\left.F_{T}^{s p}=F_{R}^{s p}=0\right)$, the systems synchronize identically for coupling strengths $c \geq 0.1$, as shown by numerical simulations of Eq. (1) and Eq. (2) as well as computations of the transverse Lyapunov exponents [23].

In an experiment, the transmitter and receiver systems are inevitably different from each other. In this situation, identical synchronization is not a mathematical solution of the model equations. Nonetheless, the deviation from the identity solution can be small over some range of parameters. An evaluation of the effect of parameter mismatches between the transmitter and the receiver on the quality of synchronization in the presence of a realistic level of spontaneous emission noise showed that the error is tolerable for a $5 \%$ or so mismatch at $c=1$, whereas for $c=0.4$ the error is large for almost all mismatches [23]. Thus, as one would expect, the open loop conifguration $(c=$ 1 ) is most robust with respect to parameter mismatch. This conclusion was confirmed experimentally [24], where approximate identical synchronization was found for a range of coupling strengths $c$ with a minimum synchronization

\footnotetext{
${ }^{1}$ The dimensionless gain $g(n, s)$ is obtained through an expansion of the gain function about the continuous-wave operating point of the laser, resulting in $g(n, s)=$ $1+\gamma_{\mathrm{n}} /\left(\mathcal{J} \gamma_{\mathrm{s}}\right) n-\gamma_{\mathrm{p}} / \gamma_{\mathrm{c}} s$. All parameters of Eq. (1), Eq. (2), and the gain function can be measured experimentally [25] and the values used are found in [23].
} 
a)

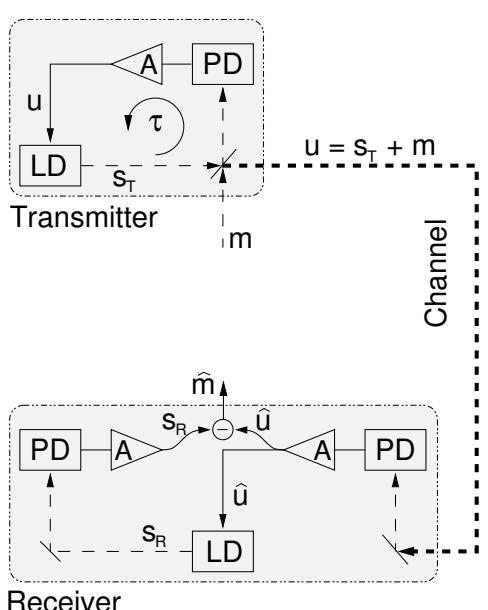

b)

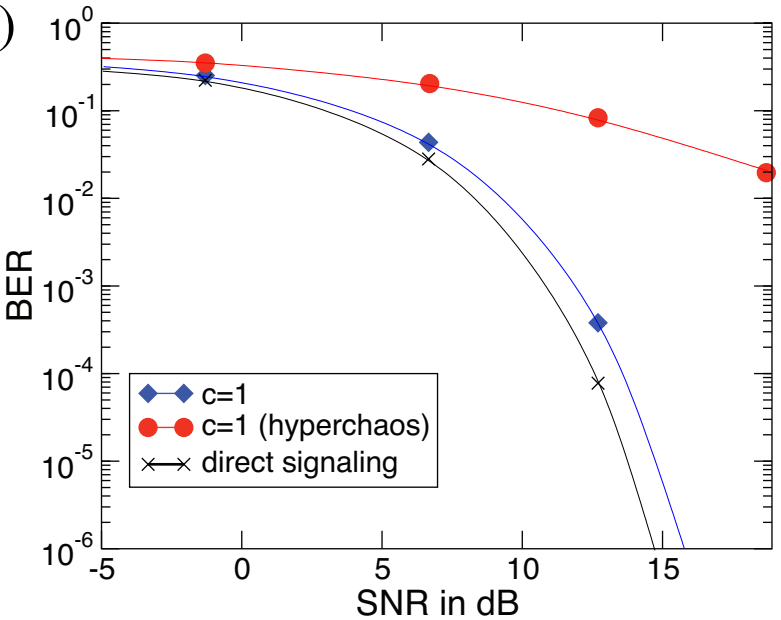

Figure 3: (a) Communication setup based on synchronized coupled optoelectronic devices in an open loop configuration $(c=1)$. (b) Numerical estimated bit error rate (BER) versus effective signal to noise ratio (SNR). Adapted from Abarbanel et al. (2001) [23].

error for the open loop configuration.

The case of two coupled optoelectronic devices is an example of a system where the noise-free model with identical transmitter and receiver predicts identical synchronization (all transverse Lyapunov exponents are negative) and where the inclusion of noise and parameter mismatches does not destroy synchronized behavior in any essential way. However, this is not true in general. For instance, the so-called phenomenon of attractor bubbling [26, 27] may occur in the presence of even small noise and small parameter mismatches may cause large distortions of the synchronization manifold and therefore large synchronization errors [28]. Although there exist criteria that guarantee high-quality synchronization [26, 29, 30], these are often either to difficult to apply or too conservative [31]. The lack of a simple and generally reliable way to determine how to couple two oscillators to achieve high-quality synchronization means that, in many cases, the computationally most efficient approach is to include realistic levels of noise and parameter mismatches in the model and check synchronization quality by direct simulation.

Synchronization leads to communication - even when the signals used are chaotic. The main idea of chaos communication schemes is to mix the message with a chaotic carrier in the transmitter and to send the mixed signal to the receiver. At the receiver, the message can be recovered by comparing 
the transmitted mixed signal to the regenerated message-free chaotic carrier. Indeed, under ideal conditions (e.g. no noise) it is possible to achieve perfect synchronization and message recovery in chaos communication schemes that utilize chaos modulation to encode and decode information $[14,15,16,17,32$, 33]. The key idea of this approach is to inject the information signal $m(t)$ into the feedback loop of the chaotic transmitter system, as depicted in Fig. 3a. The feedback is selected in such a way that the remaining subsystem is conditionally stable. In this case, the signal $u(t)=s_{T}(t)+m(t)$ returns back to the nonlinear laser diode and drives the dynamics of the transmitter. The signal $u(t)$ is also transmitted to the receiver, where it drives the dynamics and generates oscillations of the receiver system that are identical to the chaotic oscillations in the transmitter, i.e. $\hat{u}=u$ and therefore $s_{R}=s_{T}$. The message can then be recovered using signal subtraction (as shown in Fig. 3a) and, in the absence of noise, the information can be restored exactly after initial transients $(\hat{m}=m)$.

All practical communication channels introduce signal distortions that alter the chaotic waveform shape, resulting in received chaotic oscillations that do not precisely represent the transmitted oscillations. Channel noise, filtering, attenuation variability and other distortions in the channel corrupt the chaotic carrier and information signal. The presence of these channel distortions significantly hamper the onset of identical synchronization of the chaotic systems and imperfect synchronization results in information loss.

As an example, we considered information loss due to white Gaussian channel noise for the communication system shown in Fig. 3a [23]. Fig. 3b summarizes the information loss by showing the bit error rate (BER) versus the signal to noise ratio (SNR), where the latter is defined as the energy per bit divided by the power spectral density of the noise. Results are shown of calculations for the open loop configuration $(c=1)$, and, as a reference, the case of "direct signaling", where it is assumed that the receiver has a perfect copy of the transmitter's chaotic carrier signal. It is seen that one of the chaos-communication BER curves (diamond-symbol) is close to the direct-signaling curve, indicating good synchronization of transmitter and receiver despite the noisy channel. This case corresponds to a situation where the device dynamics is in a regime of low-dimensional chaos, i.e. the Lyapunov dimension $D_{L}$ of the chaotic attractor is in the range of three to four. In contrast, the chaos-communication BER curve shown with circle symbols in Fig. 3b is far to the right of the direct-signaling curve, implying substantial information losses due to noise-induced desynchronization events. 
This curve corresponds to the case of high-dimensional chaos $\left(D_{L}>5\right)$. Nevertheless, even for the case of high-dimensional chaos, the noise entering the feedback loop does not cause global desynchronization and thus it is still possible to communicate. This result demonstrates the structural stability of the synchronized state.

It is true in general that BER performance curves for synchronizationbased chaos communication lie to the right of the curve for direct signaling for any non-zero coupling. A fundamental reason for performance losses of synchronization-based chaos communication schemes as compared to traditional schemes is that chaotic systems continuously generate non-redundant information and have a positive Kolmogorov-Sinai entropy. In contrast, traditional schemes, most of which are based on periodic signals and carriers that are generated by stable systems, are characterized by a zero KolmogorovSinai entropy, which means that without any input the average speed of non-redundant information generation is zero. Therefore, even in an ideal environment, in order for two chaotic systems to remain perfectly synchronized one must transmit an amount of information per unit time that is equal or larger than the Kolmogorov-Sinai entropy [34]. As a result, the performance curve of a chaotic communication scheme is shifted to the right. In an non-ideal environment, further performance degradation may occur when the transmitter-receiver system temporarily loses synchronization due to extreme channel noise events. To re-establish synchrony more information per unit time is needed than that specified by the Kolmogorov-Sinai entropy and periods of desynchronization lead to information loss that is in addition to the information loss that results from channel-noise induced bit flips. However, in many cases the degradation caused by nonzero KolmogorovSinai entropy is insignificant, as for example for the optoelectronic systems in Fig. 3 where the chaos-communication curve (diamond-symbol) is quite close to the direct-signaling curve that assumes the receiver has a perfect copy of the transmitter's chaotic carrier signal.

In the example above, information is encoded in the signal amplitude and no error correction codes are applied. In this context, reliable communication (low BER) can be achieved only for low noise levels in the communication channel even for the direct signaling case, which requires no chaos synchronization. The potentially significant additional information losses for chaos communication schemes that result because of imperfect synchronization can be minimized through a careful choice of the chaotic carrier signal. Furthermore, the levels of channel-noise that are required for reliable optical com- 
munication can be straightforwardly achieved in bench-top experiments and in communication applications that use optical fiber links. For the system shown in Fig. 3a, a data rate of 2.5 Gbit/s was achieved in a bench-top experiment by Tang and Liu [24]. Chaos communication of high-speed digital data over a commercial metropolitan area fiber-optic network was recently demonstrated by Argyris et al. [35], where the problem of dispersion was overcome by inserting appropriate lengths of dispersion-compensating fiber. This latter result demonstrates that chaos-based encryption methods can be integrated into a real-world system and operate at high data rates with reasonably low errors. In other words, in the context of high-speed optical communication networks, which are the backbone of the worldwide data transmission infrastructure, chaotic carriers offer a certain degree of intrinsic privacy in the data transmission implemented at the hardware level, which could complement both classical software-based [36] and quantum [37] cryptography systems.

\section{Wireless communication using chaotic pulse position modula- tion}

Compared to the situation where communication takes place through lownoise optical fibers, signal deterioration in wireless communication is much more significant. A wireless communication channel is noisy, introduces significant signal distortions, and exhibits effects due to multipath propagation. For this reason, many of the early chaotic communication schemes are impractical for wireless applications because they have performance characteristics much worse than those of traditional schemes such as binary phase shift keying and binary frequency shift keying modulations [38]. In contrast, many recently suggested chaos communication schemes have bit-error-rate characteristics that are very close to the ideal ones $[6,8,39,40,41,42]$ and offer performance advantages over conventional narrowband communications schemes in multipath environments [43] because of their inherent broadband nature. Chaos communication schemes are therefore a viable alternative to traditional approaches.

One of the recently suggested ways to minimize the degradation of performance due to channel noise, distortions, and interference is to use communication systems based on chaotic pulse position modulation (CPPM). In the chaotic pulse position modulation system a sequence of identically shaped pulses with chaotic time intervals between them replaces continuous chaotic 
waveforms as an information carrier [8, 39]. The information is encoded onto the pulse train by altering the time position of transmitted pulses with respect to the chaotic carrier. Since the information about the state of the chaotic system is contained entirely in the timing between pulses, the distortions that affect the pulse shape do not significantly influence the ability of the chaotic pulse generators to synchronize. Although it was found that CPPM performs slightly worse than binary phase shift keying in a additive white Gaussian noise channel, this wideband system provides several advantages: It exhibits self-synchronization, thus eliminating the need for special synchronizing sequences, is characterized by a low probability of intercept and low probability of detection ${ }^{2}$, improves privacy and there exist multiplexing strategies that allow several users to communicate simultaneously over the same channel $[8,9,39]$.

The ability of this self-synchronizing CPPM method to communicate not only over a Gaussian noise channel but in the presence of significant nonstationary signal distortions has been studied experimentally using a freespace laser communication link [44].

A schematic representation of the chaotic free-space laser communication system is shown in Fig. 4a. CPPM Tx and CPPM Rx represent the analog circuit implementation of the CPPM transceiver. The optical part of the experiment consisted of an intensity-modulated semiconductor laser beam $(10 \mathrm{~mW}, \lambda=690 \mathrm{~nm})$ that propagated by means of a single-mode fiber, a lens relay system and a telescope (Celestrone) to a $45^{\circ}$ roof-mounted mirror. The light subsequently traveled over an atmospheric path of length $L=2.5 \mathrm{~km}$ to a 4" corner cube mounted on top of a water tower, where it was reflected and propagated back to the roof-mounted mirror. The receiver system used the same telescope and lens relay system as the transmitter. The total doublepass atmospheric laser beam propagation distance was approximately $L \approx$ $5 \mathrm{~km}$.

Figure $4 \mathrm{~b}$ illustrates the severe pulse amplitude fluctuations of the signal entering the CPPM demodulator caused by atmospheric turbulence. It also shows that the pulse propagation time $\tau_{m}$, which is measured between the leading front of the TTL pulse applied to the laser and the maximal point

\footnotetext{
${ }^{2}$ As compared to pulse-position modulation schemes using digitally generated repeating sequences of pseudo-random bits, data transmission via CPPM avoids spectral peaks associate with the repetition of the pseudo-random sequence and time-digitization. It is therefore harder to detect.
} 
(a)

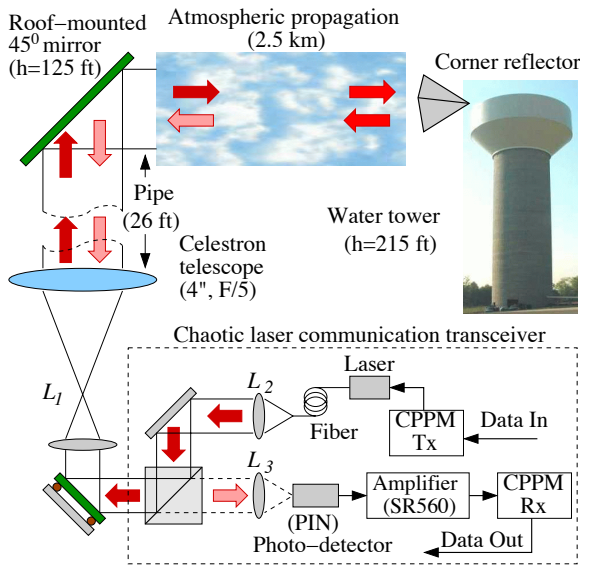

(b)

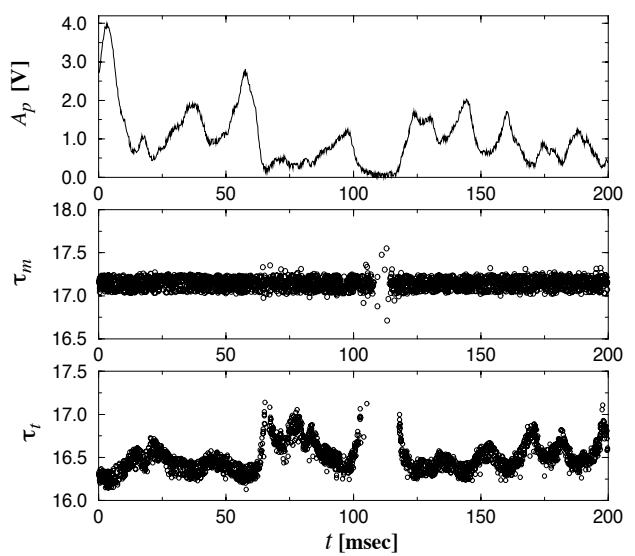

Figure 4: Robust chaos communication over a free-space laser link. (a) Experimental setup. (b) Fluctuations of the CPPM pulses after traveling through atmospheric turbulence. Pulse amplitude $A_{p}$ measured in volts at the output of the amplifier - (top). Propagation times $\tau_{m}$ - (middle) and $\tau_{t}$ - (bottom). Adapted from Rulkov et al. (2002) [44].

of the received pulse, varies only within a $0.2 \mu$ s time interval. However, in order to trigger the CPPM demodulator circuit, the received pulse amplitude has to exceed a certain threshold level. Therefore, the actual delay time $\tau_{t}$, as seen by the transceiver, depends on the amplitude of the received pulses and fluctuates (see Fig. 4, bottom). Nevertheless, the variations of the pulse propagation time are small enough for the CPPM controller to self-synchronize and to maintain the stability of the communication link.

The gaps visible in Fig. 4b are caused by pulse amplitude fading when the pulse amplitude falls to the photo-receiver noise level, which is below the threshold level. The gaps are audible as occasional clicks when using the free-space laser communication system for real-time voice communication. They are the main contribution to the total BER of $1.92 \times 10^{-2}$ measured in this experiment. Indeed, errors that are not related to the complete failure of the channel because of fading instances contributed to the BER only $\sim 5.5 \times$ $10^{-5}$. Thus, chaos communication using pulses and encoding information in the timing of the pulses appears to be a particularly robust communication scheme. 


\section{Conclusion}

Communication systems based on chaos and nonlinear dynamics are still in their infancy, although astonishing progress has been made in the recent years. Within less than two decade chaos communication developed from an idea to a communication technology that can now be compared in a meaningful way to traditional communication approaches. Optical communication with gigabit-per-second transmission rates and very low bit-error rates, comparable to those of traditional communication systems, has been achieved and wireless communication schemes have come close to ideal bit-error rate performances. The simplicity and ease of control of many of these nontraditional systems makes it easy to envision practical applications such as cost-effective private communication.

Another merit of research on chaos communication is that it challenges us to think about fundamental issues of information transmission. This research extends known communication techniques beyond the area of regular and repeating signals and shows that parties can communicate equally well using irregular signals and pulse sequences that never repeat exactly. This is relevant for biological systems, where non-sinusoidal, pulse-like waveforms are used to convey information.

\section{Acknowledgments}

I gratefully acknowledge the long-term financial support of the US Army Research Office. I am deeply indebted to my advisor H. Abarbanel and the many researchers at the Institute for Nonlinear Science at UCSD who together with their UCLA colleagues were the driving force behind the presented research on chaos communication. In particular I want to thank L. Kocarev, N. Rulkov, M. B. Kennel, L. Tsimring, S. Tang, and J.M. Liu as well as U. Parlitz and D. Gauthier for numerous discussions on the subject.

\section{References}

[1] E. Ott, C. Grebogi, J. A. Yorke, Controlling chaos, Phys. Rev. Lett. 64 (1990) $1196-1199$.

[2] H. Fujisaka, T. Yamada, Stability theory of synchronized motion in coupled-oscillator systems, Prog. Theor. Phys. 69 (1983) $32-47$. 
[3] V. S. Afraimovich, N. N. Verichev, M. I. Rabinovich, Stochastic synchronization of oscillation in dissipative systems., Radiophysics and Quantum Electronics (English Translation of Izvestiya Vysshikh Uchebnykh Zavedenii, Radiofizika) 29 (1986) 795 - 803.

[4] L. M. Pecora, T. L. Carroll, Synchronization in chaotic systems, Phys. Rev. Lett. 64 (1990) $821-824$.

[5] B. Sklar, Digital communications : fundamentals and applications, Prentice-Hall, Englewood Cliffs, NJ, 1988.

[6] G. M. Maggio, N. Rulkov, L. Reggiani, Pseudo-chaotic time hopping for uwb impulse radio, IEEE T. Circuits-I. 48 (12) (2001) 1424-1435.

[7] C. C. Chen, K. Yao, K. Umeno, E. Biglieri, Design of spread-spectrum sequences using chaotic dynamical systems and ergodic theory, IEEE T. Circuits-I. 48 (9) (2001) 1110-1114.

[8] N. F. Rulkov, M. M. Sushchik, L. S. Tsimring, A. R. Volkovskii, Digital communication using chaotic-pulse-position modulation, IEEE T. Circuits-I. 48 (12) (2001) 1436-1444.

[9] L. E. Larson, J.-M. Liu, L. S. Tsimring (Eds.), Digital Communications Using Chaos and Nonlinear Dynamics, Institute for Nonlinear Science, Springer, 2006.

[10] G. Kolumban, G. Kis, Z. Jákó, M. P. Kennedy, FM-DCSK: a robust modulation scheme for chaotic communications, IEICE Trans. Fundamentals of Electronics, Communication, and Computer Science E81A(9) (1998) $1798-8002$.

[11] S. Hayes, C. Grebogi, E. Ott, Communication with chaos, Phys. Rev. Lett. 70 (1993) $3031-3034$.

[12] S. Hayes, C. Grebogi, E. Ott, A. Mark, Experimental control of chaos for communication, Phys. Rev. Lett. 73 (1994) 1781 - 1784.

[13] N. J. Corron, S. D. Pethel, K. Myneni, Synchronizing the information content of a chaotic map and flow via symbolic dynamics, Phys. Rev. E 66 (2002) 036204/1-5. 
[14] D. R. Frey, Chaotic digital encoding: an approach to secure communication, IEEE Trans. Circuits Syst. 40 (1993) 660 - 666.

[15] A. R. Volkovskii, N. F. Rulkov, Synchronous chaotic response of a nonlinear oscillator system as a principle for the detection of the information component of chaos, Tech. Phys. Lett. 19 (1993) $97-99$.

[16] L. Kocarev, U. Parlitz, General approach for chaotic aynchronization with applications to communication, Phys. Rev. Lett. 74 (1995) 5028 5031 .

[17] U. Feldmann, M. Hasler, W. Schwarz, Communication by chaotic signals: the inverse system approach, Int. J. Ciruit Theory Appl. 24 (1996) $551-579$.

[18] L. Kocarev, K. S. Halle, K. Eckert, L. O. Chua, U. Parlitz, Experimental demonstration of secure communications via chaotic synchronization, Int. J. Bifurcation and Chaos 2 (1992) $709-713$.

[19] K. M. Cuomo, A. V. Oppenheim, Circuit implementation of synchronized chaos with applications to communications, Phys. Rev. Lett. 71 (1993) $65-68$.

[20] G. D. VanWiggeren, R. Roy, Communication with chaotic lasers, Science 279 (5354) (1998) 1198-1200.

[21] G. VanWiggeren, R. Roy, Optical communication with chaotic waveforms, Phys. Rev. Lett. 81 (1998) 3547 - 3550.

[22] J. P. Goedgebuer, L. Larger, H. Porte, Optical cryptosystem based on synchronization of hyperchaos generated by a delayed feedback tunable laser diode, Phys. Rev. Lett. 80 (1998) 2249 - 2252.

[23] H. D. I. Abarbanel, M. B. Kennel, L. Illing, S. Tang, H. F. Chen, J. M. Liu, Synchronization and communication using semiconductor lasers with optoelectronic feedback, IEEE J. Quantum. Electron. 37 (10) (2001) 1301-1311.

[24] S. Tang, J. M. Liu, Message encoding-decoding at 2.5 Gbits/s through synchronization of chaotic pulsing semiconductor lasers, Opt. Lett. 26 (2001) $1843-1845$. 
[25] T. B. Simpson, J. M. Liu, A. Gavrielides, V. Kovanis, P. M. Alsing, Period-doubling route to chaos in a semiconductor laser subject to optical injection, Appl. Phys. Lett. 64 (1994) 3539 - 3541.

[26] P. Ashwin, J. Buescu, I. Stewart, Bubbling of attractors and synchronization of chaotic oscillators, Phys. Lett. A 193 (1994) 126 - 139.

[27] P. Ashwin, J. Buescu, I. Stewart, From attractor to chaotic saddle: a tale of transverse instability, Nonlinearity 9 (1996) $703-737$.

[28] L. Illing, J. Brocker, L. Kocarev, U. Parlitz, H. D. I. Abarbanel, When are synchronization errors small?, Phys. Rev. E 66 (3) (2002) 036229.

[29] D. J. Gauthier, J. C. Bienfang, Intermittent loss of synchronization in coupled chaotic oscillators: Toward a new criterion for high-quality synchronization, Phys. Rev. Lett. 77 (1996) 1751 - 1754.

[30] R. Brown, N. Rulkov, Synchronization of chaotic systems: Transverse stability of trajectories in invariant manifolds, Chaos 7 (1997) $395-413$.

[31] J. N. Blakely, D. J. Gauthier, Attractor bubbling in coupled hyperchaotic oscillators, Int. J. Bifurcation and Chaos 10 (2000) 835 - 847.

[32] K. S. Halle, C. W. Wu, M. Itoh, L. O. Chua, Spread spectrum communication through modulation of chaos, Int. J. Bifurcation Chaos 3 (1993) $469-477$.

[33] U. Parlitz, L. Kocarev, T. Stojanovski, H. Preckel, Encoding messages using chaotic synchronization, Phys. Rev. E 53 (1996) 4351 - 4361.

[34] T. Stojanovski, L. Kocarev, R. Harris, Applications of symbolic dynamics in chaos synchronization, IEEE T. Circuits-I. 44 (10) (1997) $1014-1018$.

[35] A. Argyris, D. Syvridis, L. Larger, V. Annovazzi-Lodi, P. Colet, I. Fischer, J. Garcia-Ojalvo, C. R. Mirasso, L. Pesquera, K. A. Shore, Chaosbased communications at high bit rates using commercial fibre-optic links, Nature 438 (2005) 343-346.

[36] C. E. Shannon, Communication theory of secrecy systems, Bell Syst. Technic. J. 28 (4) (1949) $656-715$. 
[37] C. Kurtsiefer, P. Zarda, M. Halder, H. Weinfurter, P. M. Gorman, P. R. Tapster, J. G. Rarity, Quantum cryptography: A step towards global key distribution, Nature 419 (6906) (2002) 450-450.

[38] C. C. Chen, K. Yao, Stochastic-calculus-based numerical evaluation and performance analysis of chaotic communication systems, IEEE T. Circuits-I. 47 (12) (2000) 1663-1672.

[39] M. Sushchik, N. Rulkov, L. Larson, L. Tsimring, H. Abarbanel, K. Yao, A. Volkovskii, Chaotic pulse position modulation: A robust method of communicating with chaos, IEEE Commun. Lett. 4 (4) (2000) 128-130.

[40] Z. Galias, G. M. Maggio, Quadrature chaos-shift keying: Theory and performance analysis, IEEE T. Circuits-I. 48 (12) (2001) 1510-1519.

[41] G. M. Maggio, Z. Galias, Applications of symbolic dynamics to differential chaos shift keying, IEEE T. Circuits-I. 49 (12) (2002) 1729-1735.

[42] A. R. Volkovskii, L. S. Tsimring, N. F. Rulkov, I. Langmore, Spread spectrum communication system with chaotic frequency modulation, Chaos 15 (3) (2005) 033101.

[43] G. Kolumban, M. P. Kennedy, G. Kis, Z. Jákó, Chaotic communications with correlator receivers: Theory and performance limits, Proc. IEEE 90 (2002) 711.

[44] N. F. Rulkov, M. A. Vorontsov, L. Illing, Chaotic free-space laser communication over a turbulent channel, Phys. Rev. Lett. 89 (2002) $277905 / 1-4$. 